\title{
GaN Films under Different Growth Mechanisms Studied by Synchrotron X-ray Absorption Spectroscopy
}

Qing-Xuan LII,a , Yu-Jia LIU ${ }^{1, b}$, Yi LIANG ${ }^{1, c}$, Hao-Hsiung LIN ${ }^{2, d}$, Jyh-Fu Lee $^{3, e}, \mathrm{Na}$ $\mathrm{Lu}^{4, \mathrm{f}}$,Ian T. Ferguson ${ }^{5, \mathrm{~g}}$, Ling-Yu WAN ${ }^{1, \mathrm{~h}}$, Zhe Chuan Feng ${ }^{1, \mathrm{i},{ }^{*}}$

${ }^{1}$ College of Physics Science \& Technology, Guangxi Key Laboratory for the Relativistic Astrophysics, Laboratory ofoptoelectronic materials\& detection technology, Guangxi University, Nanning530004, China

${ }^{2}$ Graduate Institute of Photonics \& Optoelectronics and Department of Electrical Engineering, National Taiwan University, Taipei, 106-17, Taiwan

${ }^{3}$ National Synchrotron Radiation Research Center, Hsinchu, 300-76, Taiwan

${ }^{4}$ Sustainable Materials and Renewable Technologies Lab, Lyles school of civil engineering, Purdue University, West Lafayette, IN 47907, USA

${ }^{5}$ Department of Electrical and Computer Engineering, Missouri University of Science \& Technology, Rolla, MO 65409, USA

aliqingxuan00544@foxmail.com, b879793274@qq.com,"liangyi@gxu.edu.cn,

dhhlin@ntu.edu.tw, ${ }^{\mathrm{e}}$ jflee@nsrrc.org.tw, ${ }^{\mathrm{f}}$ luna@purdue.edu, ${ }^{\mathrm{I}}$ ianf@mst.edu, ${ }^{\mathrm{h}}$ wanlingyu75@126.com, ifengzc@gxu.edu.cn

*fengzc@gxu.edu.cn

Key words: synchrotron radiation X-ray absorption, MOCVD, MBE, GaN, residual strain

Abstract: Synchrotron radiation X-ray absorptionmeasurements wereperformed to study the structure of Si-doped n-type GaN layers grown on sapphire by metalorganic chemical vapor deposition (MOCVD) and undoped GaN epitaxial layers grown on Si by molecular beam epitaxy (MBE).It was found that the anisotropic characteristic of p-orbital from GaN/sapphire grown by MOCVD is somehow larger than GaN/Si grown by MBE.In addition, values of the lattice constant of different GaN films were deduced from the interatomic distances in the second coordination shell around Ga byextended X-ray absorption fine structure (EXAFS) analysis and the residual strain of the films was then deduced. The calculation results shows that the strain type is different in the two sets of samples which is due to the different substrate and AlN buffer layer.

\section{Introduction}

In recent years, $\mathrm{GaN}$ and related materials haveenabledmanyapplicationssuch as light emitting diodes (LED), blue/UV semiconductor lasers, high power electronic devices and solar cells[1,2]. Since GaN is an excellent piezoelectric material, a small stress can offer a significant contribution to their physical properties and the performance of $\mathrm{GaN}$ based optoelectronic devices [3,4]. However, most $\mathrm{GaN}$ is grown on sapphire, silicon or $\mathrm{SiC}$ substrates, which usually results inlarge biaxial stress in the epitaxial layers due to the differentthermal expansion coefficients and lattice constantsbetween these layers and the substrate. The stress is compressive for GaN grown on sapphire, whilethe stress is opposite for $\mathrm{GaN}$ deposited on $6 \mathrm{H}-\mathrm{SiC}$ and $\mathrm{Si}[5]$.Sucha residual stress inevitably exists in most $\mathrm{GaN}$ hetero-epitaxial layersand couldlimit the applications of GaN inoptoelectronic devices. Si-doping is normally used to form n-type $\mathrm{GaN}$ and highly Si doped $\mathrm{GaN}$ is expected to form low resistance n-type conductive layers in nitride-based LEDs, laser, and optoelectronic devices[6].It has beenreported [7] that a generation of misfit dislocationsoccur at GaN hetero-structure and stress is relaxed through the $\mathrm{Si}$-doping in GaN. It is also found that incorporation of silicon in GaN can improve the layer quality with a small stress [8]. Strain engineering seemsa key for growth of high quality GaN on non-nativesubstrates.

Synchrotron radiation (SR) technology is an advanced and powerful tool to characterize wide gap 
semiconductor materials and to allowinvestigation into atomic level. We have in recent years employedSR X-ray absorption spectroscopy (XAS) to investigate different materials, such as SiC, CdZnTe, MgZnO, AlGaN, etc. [9-12]. In this paper, we perform a SRextended X-ray absorption fine structure (EXAFS) investigation for GaN thin films grown on sapphire and on Si. The bond length around the $\mathrm{Ga}$ atom and the strain of the $\mathrm{GaN}$ films can be obtained and to compare with Raman measurements.

\section{Experiments}

Four samples labeled as P1, P2, S1 and S2 respectively are involved in this study. P1 and P2 are two Si-doped n-type $\mathrm{GaN}$ films grown on c-sapphire at $1038{ }^{\circ} \mathrm{C}$ with the $\mathrm{GaN}$ buffer grown at about $530^{\circ} \mathrm{C}$ by MOCVD[13]. The other two un-doped GaN films were grown on (111) Si substrate with the AlN buffer by MBE [14].Growth temperature for AlN buffer was between $650^{\circ} \mathrm{C}$ and $800^{\circ} \mathrm{C}$ and the typical AlN buffer layer thickness was about $10 \mathrm{~nm}$ [14]. X-ray absorption fine-structure spectra were measured for the GaN samples at Ga K-edges in X-ray fluorescence yield mode at beamline 17C of the Synchrotron Radiation Research Center in Hsinchu, Taiwan. Polarization EXAFS were operated at $15^{\circ}$ and $90^{\circ}$, which are the angles between the sample surface normal direction and the X-ray incident direction.

\section{Results and Discussion}

The Ga K-edge near edge X-ray absorptionfine structure (NEXAFS) spectra under different X-ray incident angles of the samples P1, P2, S1, S2 are shown in Fig.1. The variation of the NEXAFS peaks under grazing incidence can be seen more clearly than that under normal incidence. This is because the information depth under the grazing incidence is smaller than the depth under the normal incidence. The spectrum with the normal incidence has more contributions from the crystalline GaN.On the contrary, the spectrum under the grazing incidence has more contributions from the amorphous GaN on the surface which will cause the difference of the NEXAFS peaks betweenthe doped and undoped samples.Comparison of NEXAFS spectra recorded at two incident angles $90^{\circ}$ and $15^{\circ}$ ismade for the MOCVD GaN/sapphire P2 at inset (1) and for MBE GaN/Si S2 at inset (2), respectively. It is seen that the anisotropic characteristic of p-orbital from GaN/sapphire by MOCVD is slightly larger than GaN/Si by MBE. A low anisotropic in wurtzite GaN might indicate more portion of polycrystalline hexagonal with random orientation or theinclusions of cubic phase.

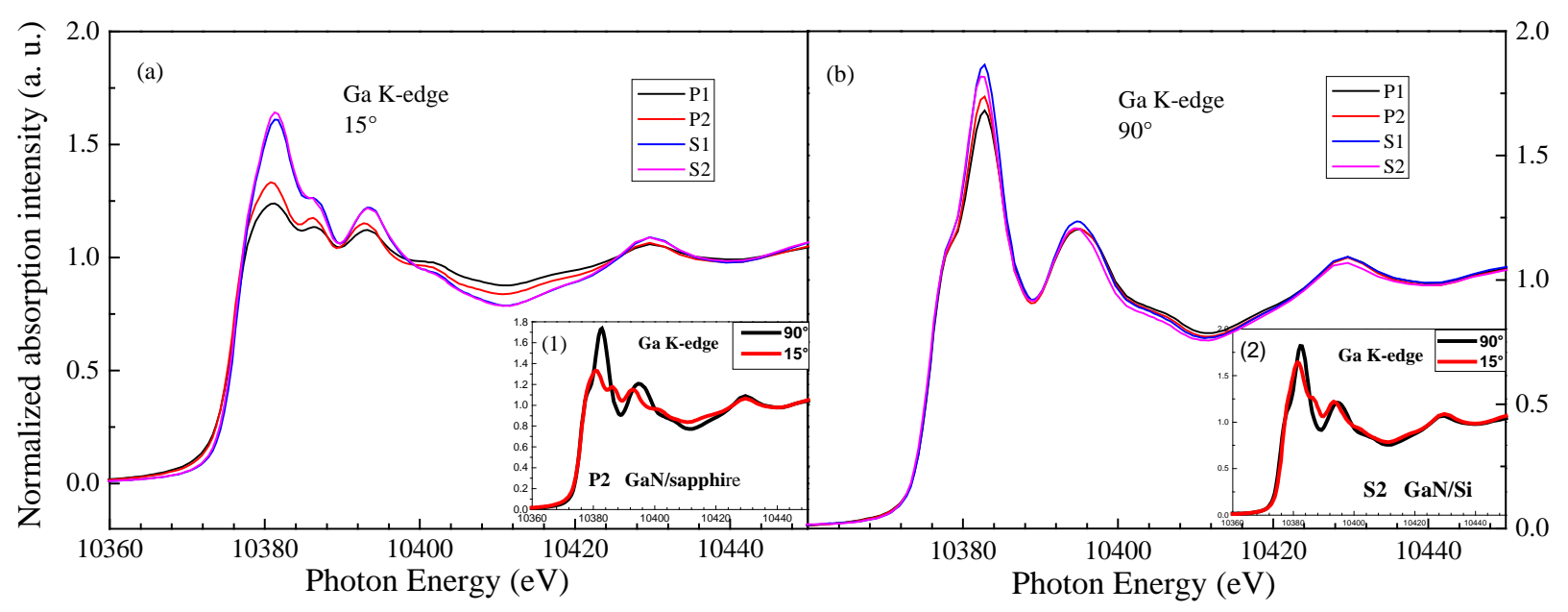

Fig.1 Ga K-edge NEXAFS spectra under (a) grazing incidence and (b) normal incidence

Polarization dependent EXAFS were performed forfour samples at the Ga K-edge. In the wurtzite structure of $\mathrm{GaN}$, the four $\mathrm{N}$ atoms in the first shell around the absorbing $\mathrm{Ga}$ atom are not at the same distance: here is a longer bond along the c-axis while the three shorter bondsare slightly inclined with 
respect to the c-plane. This leadsto the first shell XAFS having a different signal in the grazing and normal incidence of X-ray. However, the splitting in the first shell is very small, about $0.007 \AA$, and the Ga-N first shell was fitted with a single contribution. The Ga-Ga second shell is, in contrast,strongly influenced by the polarization geometry, and indeed it is the value of the second shell distances that provide us with information on the strain in the GaN epilayer since they have a simple relation to the in-plane and out-of-plane lattice parameters, a and c. In bulk GaN, six Ga atoms lie in the same (0001) basal plane as the central atom at a distance

$$
R_{G a G a}^{i n}=a .(1)
$$

And the other six Ga atoms, positioned in parallel basalplanes (three atoms above and three below), are at a distance

$$
R_{G a G a}^{\text {out }}=\sqrt{\frac{1}{3} a^{2}+\frac{1}{4} c^{2}}
$$

Under the grazingincidence of the X-ray, only the six Ga atoms above and below thebasal plane contribute to the spectrum and the measureddistance can be given by Eq. (2).The sample P1 EXAFS data fitting was performed in Fig.2 as an example using the IFEFFIT program by three steps[15]:(i) the XAFS data under grazing incidence of $\mathrm{X}$-ray is fitted to obtain the value of $\mathrm{R}_{\mathrm{GaGa}}^{\text {out }}$ for out-of-plane atoms as shown in Fig.2(a); (ii) the fit of the XAFS data under normal incidence of X-ray is performed considering a split Ga-Ga second shell. The interatomic distance of one of the two subshells is fixed to $\mathrm{R}_{\mathrm{GaGa}}^{\text {out }}$ and the other's is to obtain the in-plane Ga-Ga interatomic distance $\mathrm{R}_{\mathrm{GaGa}}^{\text {in }}$, as shown in Fig.2 (b); (iii) the value of lattice constant $a$ equals to the in-plane Ga-Ga interatomic distance $\mathrm{R}_{\mathrm{GaGa}}^{\mathrm{in}}$ and $c$ can be obtained by considering the given relation between $c$ andR $\mathrm{GaGa}_{\mathrm{Ga}}^{\text {out The }}$ fitting results are reported in Table 1.

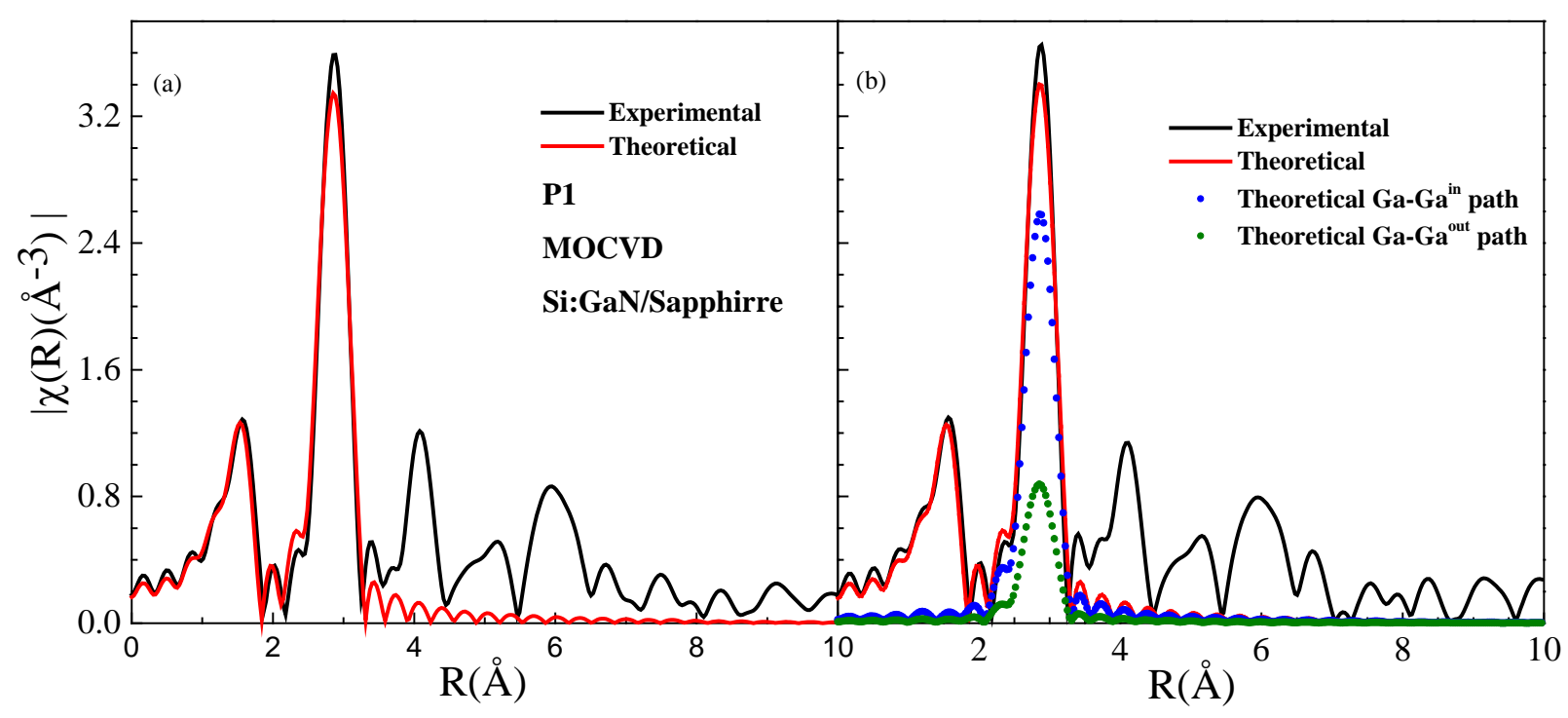

Fig.2 Fourier transformed EXAFS spectra around Ga K edge of sample P1. (a) Under grazing incidence. (b) Under normal incidence.

The lattice constantsa and $c$ can be obtained from the interatomic distances in the second coordination shell around Ga byusingEq. (1),(2) and the calculation results are listed in Table 2. The lattice constants $a_{0}$ of $3.1878 \AA$ and $c_{0}$ of $5.1850 \AA$ from relaxed, and unstrained bulk GaN were also listed in Table 2 [16].It's observed that the lattice constants of GaN grown on sapphire (P1and P2) are smaller than the unstrained bulk GaN's which means a compressive stress existing in the epitaxial films.However considering the samples S1 and S2 grown on silicon by MBE, the lattice constants of $\mathrm{S} 1$ are smaller than the unstrained bulk GaN's and the lattice constants of S2 are larger than the 
unstrained bulk GaN's. So, the strain is compressive in S1 and tensile in S2.The strain values can be quantitatively calculated by the following equations:

$\varepsilon_{x}=a / a_{0}-1,(3)$

and

$$
\varepsilon_{z}=c / c_{0}-1 .(4)
$$

The calculation results are listed in Table 2. For a convenient comparison, the values of strain deduced from Raman line shape analysis are also listed in Table 2. It is observed that the $\varepsilon_{\mathrm{x}}$ values obtained from two techniques have the same sign and arethe same order of magnitude for all the experimental samples. Moreover, the $\varepsilon_{\mathrm{x}}$ value of the same sample deduced from Raman analysis (which to be reported elsewhere) is close to the value deduced from EXAFS analysis, especially for the samples P1 and S1. It indicates that polarization EXAFS can be a useful method for calculation strain. The $\varepsilon_{\mathrm{x}}$ sign of $\mathrm{S} 1$ is different with the $\varepsilon_{\mathrm{X}}$ sign of S2, which indicates the stress type in the two samples is different with each other.It is due to the AlN interlayer and the different growing conditions.A systematic work will be carried out to investigate the effect of AlN interlayer on stress.

Table 1 Results of the quantitative XAFS analysis

\begin{tabular}{llllll}
\hline Sample & $R_{G a N}(\AA)$ & $\sigma_{G a N}^{2}\left(\AA^{2}\right)$ & $R_{G a G a}^{\text {out }}(\AA)$ & $R_{G a G a}^{\text {in }}(\AA)$ & $\sigma_{G a G a}^{2}\left(\AA^{2}\right)$ \\
\hline P1 & 1.9306 & 0.0013 & 3.1713 & 3.1825 & 0.0044 \\
P2 & 1.9329 & 0.0013 & 3.1716 & 3.1767 & 0.0044 \\
S1 & 1.9452 & 0.0037 & 3.1765 & 3.1834 & 0.0054 \\
S2 & 1.9520 & 0.0036 & 3.1833 & 3.1902 & 0.0045 \\
\hline
\end{tabular}

Table 2 Lattice constants and strain obtained from EXAFS and strain from Raman analysis

\begin{tabular}{llllll}
\hline & Bulk & P1 & P2 & S1 & S2 \\
\hline a $(\AA)$ & 3.1878 & 3.1825 & 3.1767 & 3.1834 & 3.1902 \\
c $(\AA)$ & 5.1850 & 5.1695 & 5.1750 & 5.1816 & 5.1926 \\
$\varepsilon_{\mathrm{z}}\left(\times 10^{-3}\right)$ & EXAFS & -2.99 & -1.93 & -0.66 & 1.47 \\
$\varepsilon_{\mathrm{x}}\left(\times 10^{-3}\right)$ & EXAFS & -1.67 & -3.48 & -1.38 & 0.75 \\
$\varepsilon_{\mathrm{x}}\left(10^{-3}\right)$ & Raman & -1.23 & -2.19 & -1.43 & 0.48 \\
\hline
\end{tabular}

\section{Summary}

SRX-ray absorption spectroscopy was utilized to study two sets of GaN epitaxial films grown on different substrate by different technology.Polarization-dependent near edge X-ray absorption fine structure analysisshows that GaN/sapphire grown by MOCVD has a larger anisotropic character comparing with GaN/Si grown by MBE. Bytheoretical fitting the extended X-ray absorption fine structure, the bond length of Ga-N and Ga-Ga were obtained and then values of the lattice constants and strainsfrom different GaN films were calculated. The calculation results shows that a compressive strain exists in P1, P2 and S1. A tensile strain exists in S2. These resultscorrespond wellwith the calculation results deduced from Raman scattering analysis. SR X-ray absorption spectroscopy not only can be utilized to study the structure of $\mathrm{GaN}$, but also can be used to investigate the strains.

\section{Acknowledgement}

We acknowledge the funding supportsfrom National Natural Science Foundation of China (Nos. 61367004), Guangxi Key Laboratory for the Relativistic Astrophysics-Guangxi Natural Science Creative Team funding (Nos. 2013GXNSFFA019001), and Ministry of Science and Technology 
(Nos. NSC 102-2221-E-002-191-MY3)at National Taiwan University. A number of people have improved this work by graciously providing corrections, comments, and suggestions. Special thanks to Mr.Xiaodong Jiang for all technical helps.

\section{References}

[1] Xiaoming Wu, Junlin Liu, ZhijueQuan, ChuanbingXiong, Changda Zheng, Jianli Zhang, Qinghua Mao, andFengyi Jiang. Applied Physics Letters. 104 (2014) 221101

[2] Chao Liu, YuefeiCai, Zhaojun Liu, Jun Ma, and Kei May Lau. Applied Physics Letters. 106 (2015) 181111

[3] J. W. Chen, Y. F. Chen, H. Lu,and W. J. Schaff.Applied Physics Letters.87(2005)041907

[4] N Lu, I Ferguson.Semiconductor Science and Technology 28 (2013) 074023

[5] FCWang, C L Cheng, Y F Chen, C F Huang and C C Yang. Semiconductor Science and Technology.22(2007)896-899

[6] Shuji Nakamura, Takashi Mukai, Masayuki Senoh.Journal of Applied Physics. 31 (1992) 2883

[7] Lee, I. H., Choi, I. H., Lee, C. and Shin, E. J.. Journal of Applied Physics, 83(1998) 5787-5791.

[8] Sergei Ruvimov, ZuzannaLiliental-Weber, Tadeusz Suski, Joel W. Ager III, JackWashburn,Joachim Krueger, Christian Kisielowski, Eicke R. Weber, H. Amano and I. Akasaki. Applied Physics Letters.69 (1996) 990-992

[9] Qiang Xu, Hua Yang Sun, Cheng Chen, Ling-Yun Jang, RUSLI, Suwan P. Mendis, Chin Che Tin, Zhi Ren Qiu, Zhengyun Wu, Chee Wee Liu, and ZheChuan Feng. Materials Science Forum. 717-720 (2012), 509-512

[10]Wei Zheng, Hao-Hsiung Lin, ZheChuan Feng, Fan-Hsiu Chang, Jyh-Fu Lee, Chee Wee Liu, Dong-Sing Wuu,andRui Sheng Zheng.Journal of Alloys and Compounds.582(2014) 157-160

[11]Shuchang Wang, Xiong Zhang,ZheChuan Feng and Yiping Cui. Optics Express. 22 (2014) 17440-17447

[12]DevkiTalwar, ZheChuan Feng, Jyh-Fu Lee, P. Becla. Materials Research Express. 1 (2014) 015018

[13]Z. C. Feng. Optical Engineering. 41 (2002) 2022-2031

[14]W. Tong, M. Harris, B.K. Wagner, J.W. Yu, H.C. Lin, Z.C. Feng. Surface\&Coatings Technology. 200 (2006) 3230-3234

[15]F. d'Acapito, F. Boscherini, S. Mobilio, A. Rizzi, R. Lantier. Physical Review B. 66 (2002)205411

[16] C. Kisielowski, J. Kru“ger, S. Ruvimov, T. Suski, J. W. Ager III, E. Jones, Z. Liliental-Weber, M. Rubin and E. R. Weber.Physical Review B. 54 (1996) 17745-17753 